\title{
Gravitational attraction of a vertical pyramid model of flat top-and-bottom with depth-wise parabolic density variation
}

\author{
Anand P Gokula and Rambhatla G Sastry* \\ Department of Earth Sciences, Indian Institute of Technology Roorkee, Roorkee 247 667, India. \\ *Corresponding author.e-mail: rgss1fes@iitr.ac.in; rgssastry@gmail.com
}

In 3D gravity modelling, right rectangular vertical prism model with linear and nonlinear density and polyhedral bodies with linear density variation exist in geophysical literature. Here, we propose a vertical pyramid model with depth-wise parabolic density contrast variation. Initially, we validate our analytic expression against the gravity effect of a right rectangular parallelepiped of constant density contrast. We provide two synthetic examples and a case study for illustrating the effectiveness of our pyramid model in gravity modelling. The included case study of Los Angeles basin, California demonstrates the comparative advantages of our pyramid model over a conventional right rectangular vertical prism model. Our pyramid model could be quite effective as a building block for evaluating the gravity effect of an arbitrarily-shaped 3D or 2.5-D source(s).

\section{Introduction}

Computation of theoretical gravity response of an arbitrarily-shaped 3D target body has been addressed by many authors. Different types of gravity models, like right rectangular prism, polygonal lamina and polyhedral models with constant and different depth-wise density functions have been used by several authors (Talwani and Ewing 1960; Nagy 1966; Banerjee and Das Gupta 1977; Chai and Hinze 1988; García-Abdeslem 1992, 2005; Rao et al. 1993, 1995; Pohanka 1988, 1998; Hansen 1999; Tsoulis 2000; Chakravarthi et al. 2002; Hamayun et al. 2009; Martins et al. 2010; Holstein et al. 2013; Oliveira and Barbosa 2013; D'Urso 2014a, b, 2015). Different types of density function, like exponential (Chai and Hinze 1988), parabolic (Rao et al. 1993) and hyperbolic density functions (Rao et al. 1995) have been used in sedimentary basin modelling. Chakravarthi et al. (2002) have proposed an analytical solution of right rectangular prism model with a depth-wise parabolic density contrast variation. Hansen (1999), Holstein (2003) and Hamayun et al. (2009) have used polyhedral model in gravity forward modelling with a depth-wise linear density variation.

A detailed study of singularities encountered in gravity forward modelling of different models have been addressed by Okabe (1979), Pohanka (1988), Kwok (1991), Petrović (1996), Tsoulis (2000), Tsoulis and Petrović (2001), Holstein (2002) and D'Urso (2013).

Starostenko (1978) has proposed an inhomogeneous vertical pyramid model with flat top-andbottom and sloping sides with a depth-wise linear density variation. However, he was unable to derive a complete analytical expression for its gravity effect.

Keywords. Gravity effect; vertical pyramid model with flat top-and-bottom; parabolic density variation; gravity forward modelling. 
Here, we derive the complete gravity expression for the same pyramid model with a depth-wise parabolic density contrast function variation and illustrate its effectiveness through two synthetic examples after customary validation check of our forward problem solution. We also demonstrate the usefulness and effectiveness of our model in a case study (Chai and Hinze 1988; Chakravarthi et al. 2002).

\section{Theory}

Consider an isolated regular pyramid model with depth-wise parabolic density contrast, ABCDEFGH with flat top, ABCD and bottom surface, EFGH (figure 1a). The gravity effect (figure 1b) of such a model at any arbitrary point $(x, y, z)$ in free-space is given by,

$$
\begin{aligned}
g_{\text {pyramid }}(x, y, z) & =\gamma \int_{\zeta=h_{1}}^{h_{2}} \int_{\eta=\eta_{l}}^{\eta_{u}} \int_{\xi=\xi_{l}}^{\xi_{u}} \\
& \times \frac{\Delta \rho(\zeta)(\zeta-z) d \xi d \eta d \zeta}{\left((\xi-x)^{2}+(\eta-y)^{2}+(\zeta-z)^{2}\right)^{3 / 2}},
\end{aligned}
$$

where parabolic density contrast, $\Delta \rho(\zeta)$ and limits of variables $\xi$ and $\eta$ are given by:

$$
\left.\begin{array}{l}
\Delta \rho(\zeta)=\Delta \rho_{0}^{3} /\left[\Delta \rho_{0}-\alpha\left(\zeta-h_{1}\right)\right]^{2}, \\
\xi_{l}=\left(h_{1}-\zeta\right)\left(\xi_{1}-\xi_{3}\right) /\left(h_{2}-h_{1}\right)+\xi_{1}, \\
\xi_{u}=\left(h_{1}-\zeta\right)\left(\xi_{2}-\xi_{4}\right) /\left(h_{2}-h_{1}\right)+\xi_{2}, \\
\eta_{l}=\left(h_{1}-\zeta\right)\left(\eta_{1}-\eta_{3}\right) /\left(h_{2}-h_{1}\right)+\eta_{1} \\
\eta_{u}=\left(h_{1}-\zeta\right)\left(\eta_{2}-\eta_{4}\right) /\left(h_{2}-h_{1}\right)+\eta_{2} .
\end{array}\right\}
$$

where $\gamma$ is the universal gravitational constant, $\Delta \rho_{0}$ is the density contrast observed at the ground surface in $\mathrm{g} / \mathrm{cm}^{3}, \alpha$ is a constant in $\mathrm{g} / \mathrm{cm}^{3} / \mathrm{km}$, $h_{1}$ and $h_{2}$ are the depth of the top and bottom surface of pyramid and $\zeta$ refers to depth below $h_{1}$. The value of $\alpha$ can be obtained by fitting parabolic density function (first one of equation 2) to the known density contrast-depth data of sedimentary rocks (Chakravarthi et al. 2002). $\mathrm{A}\left(\xi_{1}, \eta_{1}, h_{1}\right), \mathrm{B}\left(\xi_{1}, \eta_{2}, h_{1}\right), \quad \mathrm{C}\left(\xi_{2}, \eta_{2}, h_{1}\right), \mathrm{D}\left(\xi_{2}, \eta_{1}, h_{1}\right)$, $\mathrm{E}\left(\xi_{3}, \eta_{3}, h_{2}\right), \mathrm{F}\left(\xi_{3}, \eta_{4}, h_{2}\right), \mathrm{G}\left(\xi_{4}, \eta_{4}, h_{2}\right)$ and $\mathrm{H}\left(\xi_{4}, \eta_{3}, h_{2}\right)$ are corners of the pyramid (figure 1a). By changing the variables on right hand side (RHS) in equations (1) and (2),

$$
\xi-x=\xi^{\prime} ; \quad \eta-y=\eta^{\prime} ; \quad \zeta-z=\zeta^{\prime} .
$$

we get:

$$
\begin{aligned}
g_{\text {pyramid }}(x, y, z)= & \gamma \int_{\zeta^{\prime}=h_{1}-z}^{h_{2}-z} \int_{\eta^{\prime}=\eta_{l}^{\prime}-y}^{\eta_{u}^{\prime}-y} \int_{\xi^{\prime}=\xi_{l}^{\prime}-x}^{\xi_{u}^{\prime}-x} \\
& \times \frac{\Delta \rho\left(\zeta^{\prime}\right) \zeta^{\prime} d \xi^{\prime} d \eta^{\prime} d \zeta^{\prime}}{R^{3}},
\end{aligned}
$$

where

$$
\begin{aligned}
& \Delta \rho\left(\zeta^{\prime}\right)=\Delta \rho_{0}^{3} /\left[\Delta \rho_{0}-\alpha\left(\zeta^{\prime}+z-h_{1}\right)\right]^{2}, \\
& \xi_{l}^{\prime}=\left(h_{1}-\zeta^{\prime}-z\right)\left(\xi_{1}-\xi_{3}\right) /\left(h_{2}-h_{1}\right)+\xi_{1}, \\
& \xi_{u}^{\prime}=\left(h_{1}-\zeta^{\prime}-z\right)\left(\xi_{2}-\xi_{4}\right) /\left(h_{2}-h_{1}\right)+\xi_{2}, \\
& \eta_{l}^{\prime}=\left(h_{1}-\zeta^{\prime}-z\right)\left(\eta_{1}-\eta_{3}\right) /\left(h_{2}-h_{1}\right)+\eta_{1}, \\
& \eta_{u}^{\prime}=\left(h_{1}-\zeta^{\prime}-z\right)\left(\eta_{2}-\eta_{4}\right) /\left(h_{2}-h_{1}\right)+\eta_{2} \\
& R=\sqrt{\left(\xi^{\prime}\right)^{2}+\left(\eta^{\prime}\right)^{2}+\left(\zeta^{\prime}\right)^{2}} .
\end{aligned}
$$

Equation (3) shows the mathematical expression for pyramid model in integral form. Appendix
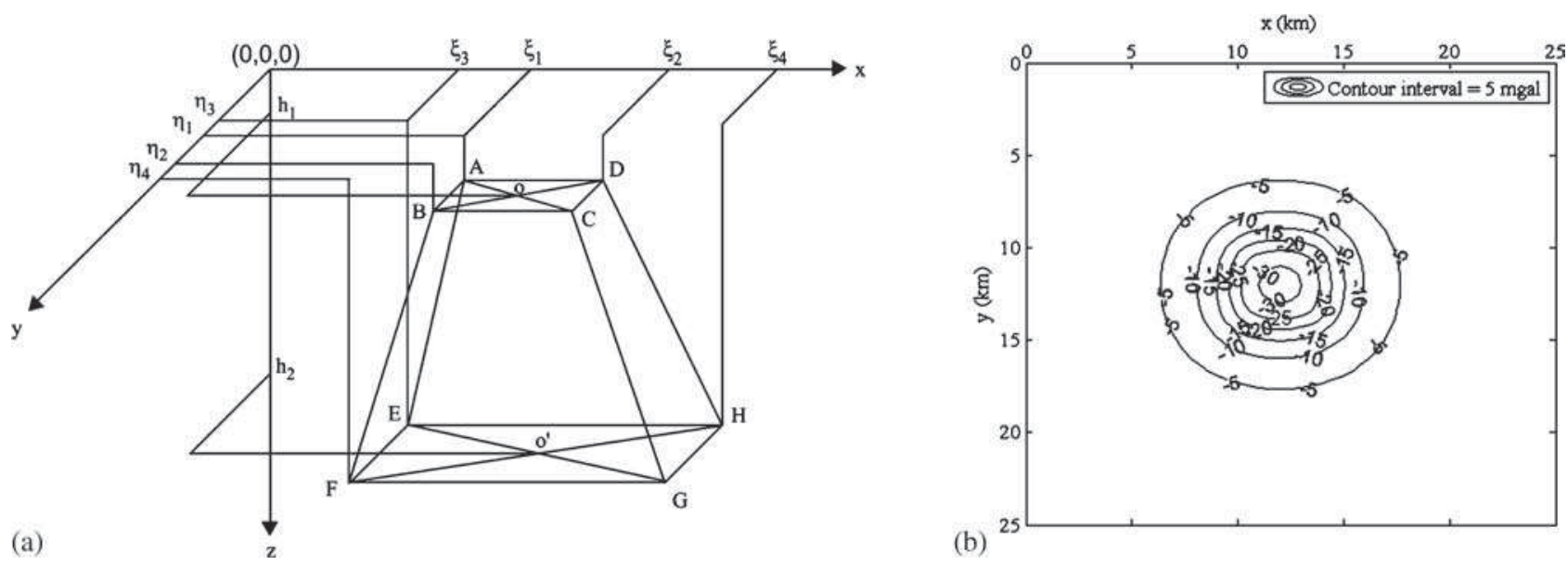

Figure 1. (a) A 3D pyramid model and its geometry with depth-wise parabolic density variation. (b) Contour plot of its gravity effect. The pyramid model parameters are as follows: $\xi_{1}=10, \xi_{2}=14, \xi_{3}=8, \xi_{4}=16, \eta_{1}=10, \eta_{2}=14, \eta_{3}=$ $8, \eta_{4}=16, \Delta \rho_{0}=-0.5206 \mathrm{~g} / \mathrm{cm}^{3}, \alpha=0.0403 \mathrm{~g} / \mathrm{cm}^{3} / \mathrm{km}, h_{1}=0.5, h_{2}=5$ and $z=0$. All length parameters and station distances are expressed in $\mathrm{km}$. 
contains the final analytical expression (forward problem solution) with relevant mathematical details. The integral evaluations on RHS of equation (3) are undertaken by Wolfram Mathematica 9.0.1. Drafting of illustrations were implemented through MATLAB 2013b.

\section{Results and discussion}

For illustration purpose, we have included two synthetic pyramid models with depth-wise parabolic density contrast and their computed gravity effects (figure 2a, b) based on equation (A5).

\subsection{Validation}

To validate our gravity forward problem solution (equation A5) for a pyramid model, we have considered a single right rectangular parallelepiped with constant density (Banerjee and Das Gupta 1977), whose gravity effect (figure 3a) is given by:

$$
\begin{gathered}
g_{z}(0,0,0)=\gamma \sigma\left[x \ln \left(y+\sqrt{x^{2}+y^{2}+z^{2}}\right)\right. \\
+y \ln \left(x+\sqrt{x^{2}+y^{2}+z^{2}}\right) \\
\left.-z \tan ^{-1} \frac{x y}{z \sqrt{x^{2}+y^{2}+z^{2}}}\right]\left.\left.\left.\right|_{x_{1}} ^{x_{2}}\right|_{y_{1}} ^{y_{2}}\right|_{z_{1}} ^{z_{2}},
\end{gathered}
$$

where $\sigma$ is the constant density of the prism in $\mathrm{g} / \mathrm{cm}^{3}$.

Accordingly, figure 3(a) corresponds to the gravity effect of a right rectangular parallelepiped (Banerjee and Das Gupta 1977) while figure 3(b) to that of our model. Our model response matches well (RMS error of $7.1 * 10^{-3} \mathrm{mgal}$ ) with that of right rectangular parallelepiped.
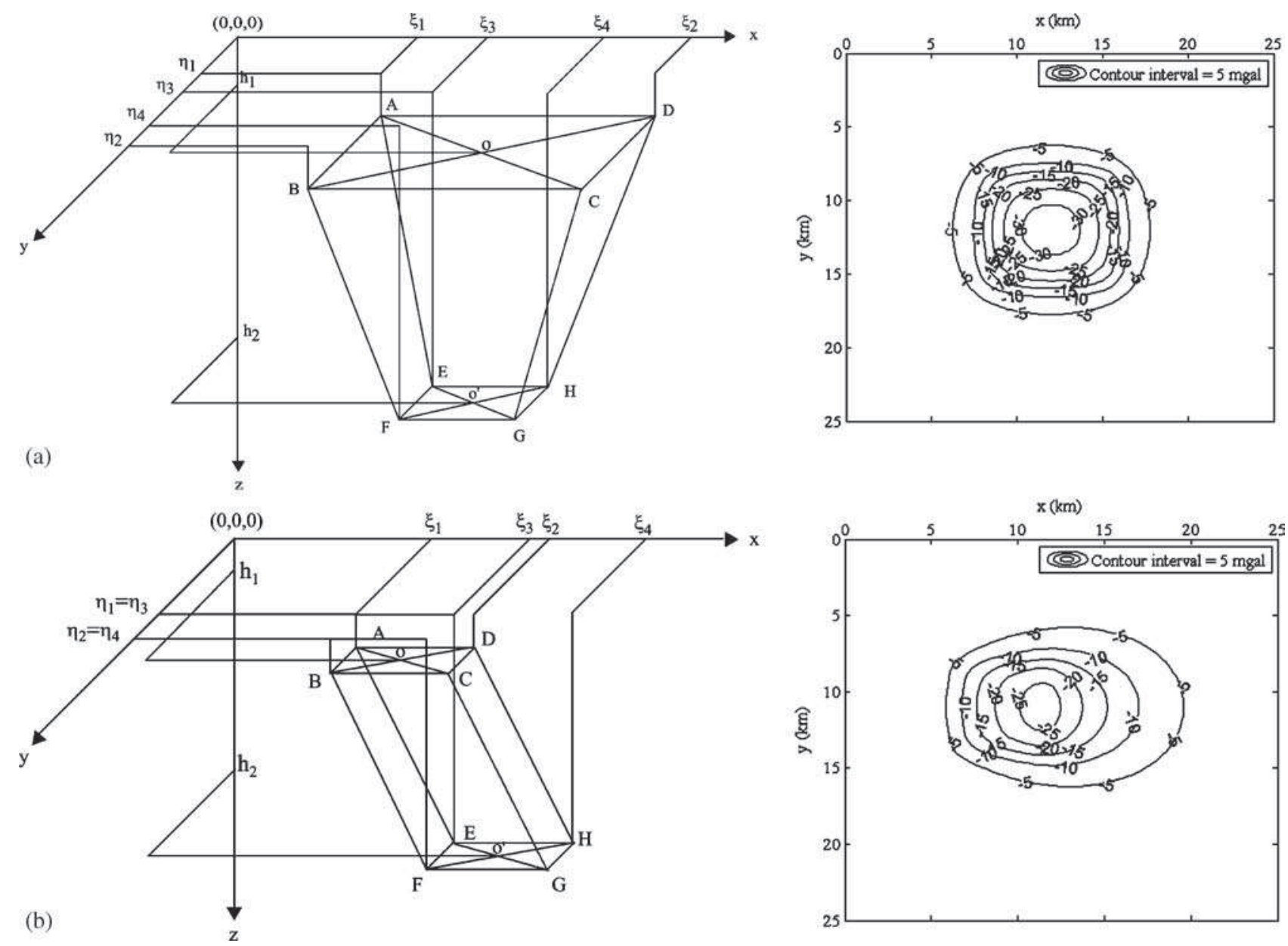

Figure 2. Two synthetic pyramid models with depth-wise parabolic density variation and their respective gravity effects. The pyramid model parameters for the two cases are as follows: (a) $\xi_{1}=8, \xi_{2}=16, \xi_{3}=10, \xi_{4}=14, \eta_{1}=8, \eta_{2}=16$, $\eta_{3}=10, \eta_{4}=14$ and $(\mathbf{b}) \xi_{1}=6, \xi_{2}=12, \xi_{3}=15, \xi_{4}=21, \eta_{1}=8, \eta_{2}=14, \eta_{3}=8, \eta_{4}=14$. Parameters, $\Delta \rho_{0}=-0.5206$ $\mathrm{g} / \mathrm{cm}^{3}, \alpha=0.0403 \mathrm{~g} / \mathrm{cm}^{3} / \mathrm{km}, h_{1}=0.5, h_{2}=5$ and $z=0$ remain same for both models. All length parameters and station distances are expressed in $\mathrm{km}$. 

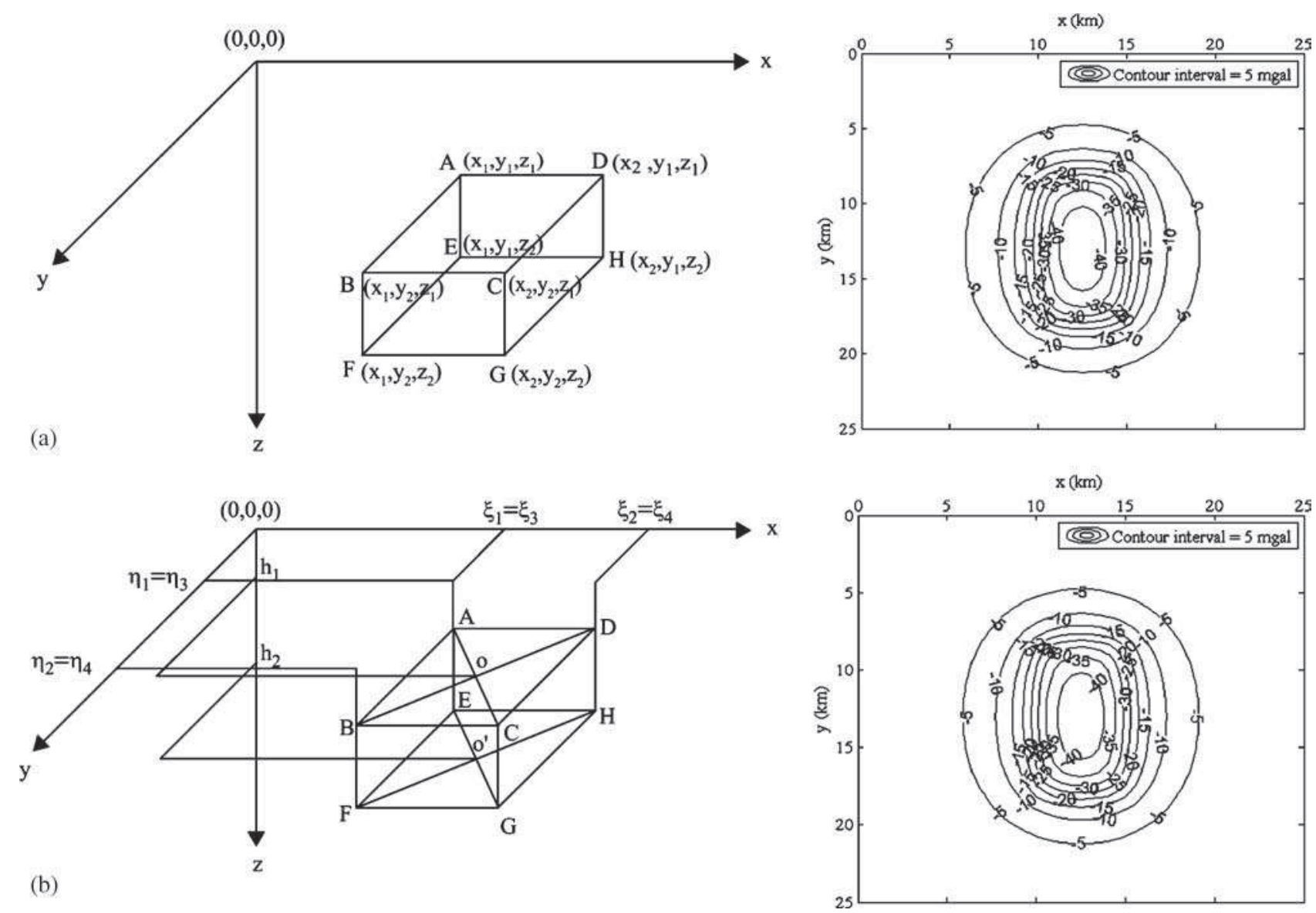

Figure 3. Validation of our forward problem solution (equation A5) through a comparison of our gravity response with that of right rectangular parallelepiped (Banerjee and Das Gupta 1977). (a) Geometry and gravity anomaly plot of right rectangular parallelepiped (Banerjee and Das Gupta 1977). The parameters for the models are as follows: $x_{1}=10, x_{2}=15$, $y_{1}=8, y_{2}=18, z_{1}=0.5, z_{2}=5$ and $\sigma=-0.5206 \mathrm{~g} / \mathrm{cm}^{3}$. (b) Geometry and gravity anomaly plot of our pyramid model. The pyramid model parameters are as follows: $\xi_{1}=10, \xi_{2}=15, \xi_{3}=10, \xi_{4}=15, \eta_{1}=8, \eta_{2}=18, \eta_{3}=8, \eta_{4}=18, h_{1}=$ $0.5, h_{2}=5, \Delta \rho_{0}=-0.5206 \mathrm{~g} / \mathrm{cm}^{3}, z=0$ and $\alpha=6 * 10^{-5}$. All length parameters and station distances are expressed in $\mathrm{km}$.

\subsection{Case study}

The case study concerns gravity modelling of Los Angeles basin, California (Chai and Hinze 1988; Chakravarthi et al. 2002). Chai and Hinze (1988) have interpreted the residual gravity field of the basin for exponential density-depth function while Chakravarthi et al. (2002) attempted it with vertical prisms of parabolic density function.

We have digitized the basement topographic map (figure 4a) (Chai and Hinze 1988), relevant residual (figure 4b) gravity (Chai and Hinze 1988) and computed gravity anomaly (figure 5a) maps (Chakravarthi et al. 2002) of Los Angeles basin on $2 \times 2 \mathrm{~km}^{2}$ grid.

Accordingly, we have carried out forward modelling of Los Angeles basin, California, using our pyramid model in which parabolic density contrast varies with depth (figure $5 \mathrm{~b}$ ). We have used the same values for input parameters, $\Delta \rho_{0}(=-0.5206$ $\left.\mathrm{g} / \mathrm{cm}^{3}\right)$ and $\alpha\left(=0.0576 \mathrm{~g} / \mathrm{cm}^{3} / \mathrm{km}\right)$ as provided by Chakravarthi et al. (2002). By treating the residual gravity anomaly of Los Angeles basin, California (Chai and Hinze 1988) as a standard map, we compare our result with that of Chakravarthi et al. (2002) in table 1. Figure 6 illustrates our model discretization with that of Chakravarthi et al. (2002).

\subsection{Discussion}

To our knowledge, gravity effect of polyhedra with parabolic density variation does not exist in geophysical literature. So, our current effort is novel in gravity forward modelling. In this regard, figure $2(a, b)$ demonstrates the flexibility of our pyramid model in gravity modelling.

Our theoretical gravity expression for a pyramid model with sloping sides is validated against that of a right rectangular parallelepiped model (Banerjee and Das Gupta 1977). In this process, we have addressed the issue of singularities that arise in the relevant analytical expressions as detailed below. 

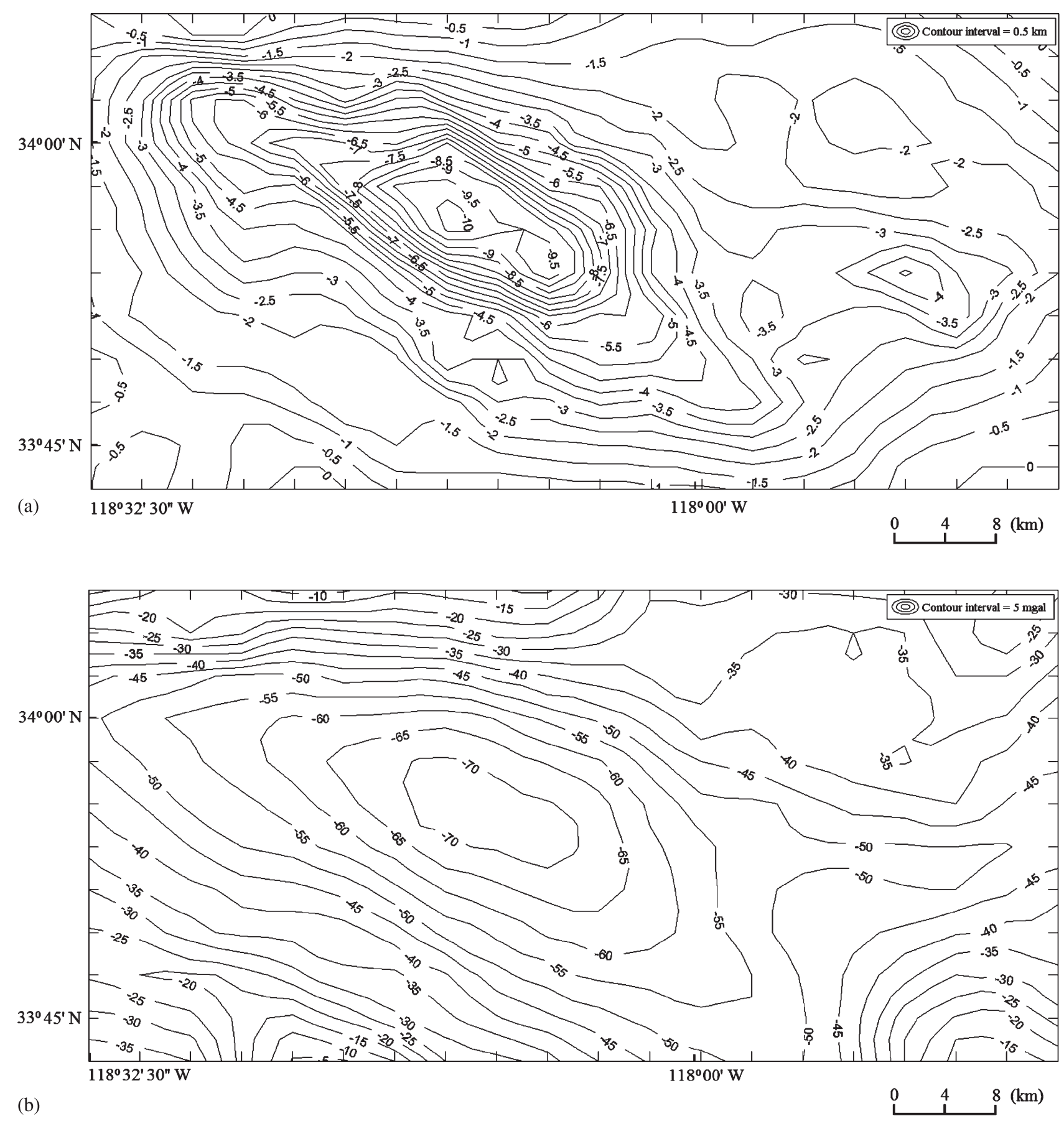

Figure 4. (a) Basement topography and (b) residual gravity anomaly map of Los Angeles basin, California (Chai and Hinze 1988).

\subsubsection{Reduction of parabolic density to constant density}

The first and fourth parts of equation (A4) is singular, when $\alpha=0$. In that case we evaluate them when $\lim _{\alpha \rightarrow 0}$ by applying conventional approach (L'Hospital's rules) twice and once for the first and fourth parts of equation (A4) respectively, which led to a root mean square (RMS) and normalized root mean square (NRMS) errors of the order of $4.18 \mathrm{mgal}$ and $0.08 \%$ respectively compared to that of right rectangular parallelepiped. But this analysis is valid only for right rectangular parallelepiped.

Our pyramid model with parabolic density function can also be converted into right rectangular parallelepiped of constant density with an RMS error of $0.5-7.1 * 10^{-3} \mathrm{mgal}$ corresponding to $\alpha$ values in the range, $6 * 10^{-5}-5 * 10^{-7} \mathrm{~g} / \mathrm{cm}^{3} / \mathrm{km}$ respectively. For all other possible positions of pyramid model with constant density function, the depth of the top surface of the pyramid model should be more than $1 / 20$ times of the unit grid station interval. It may 

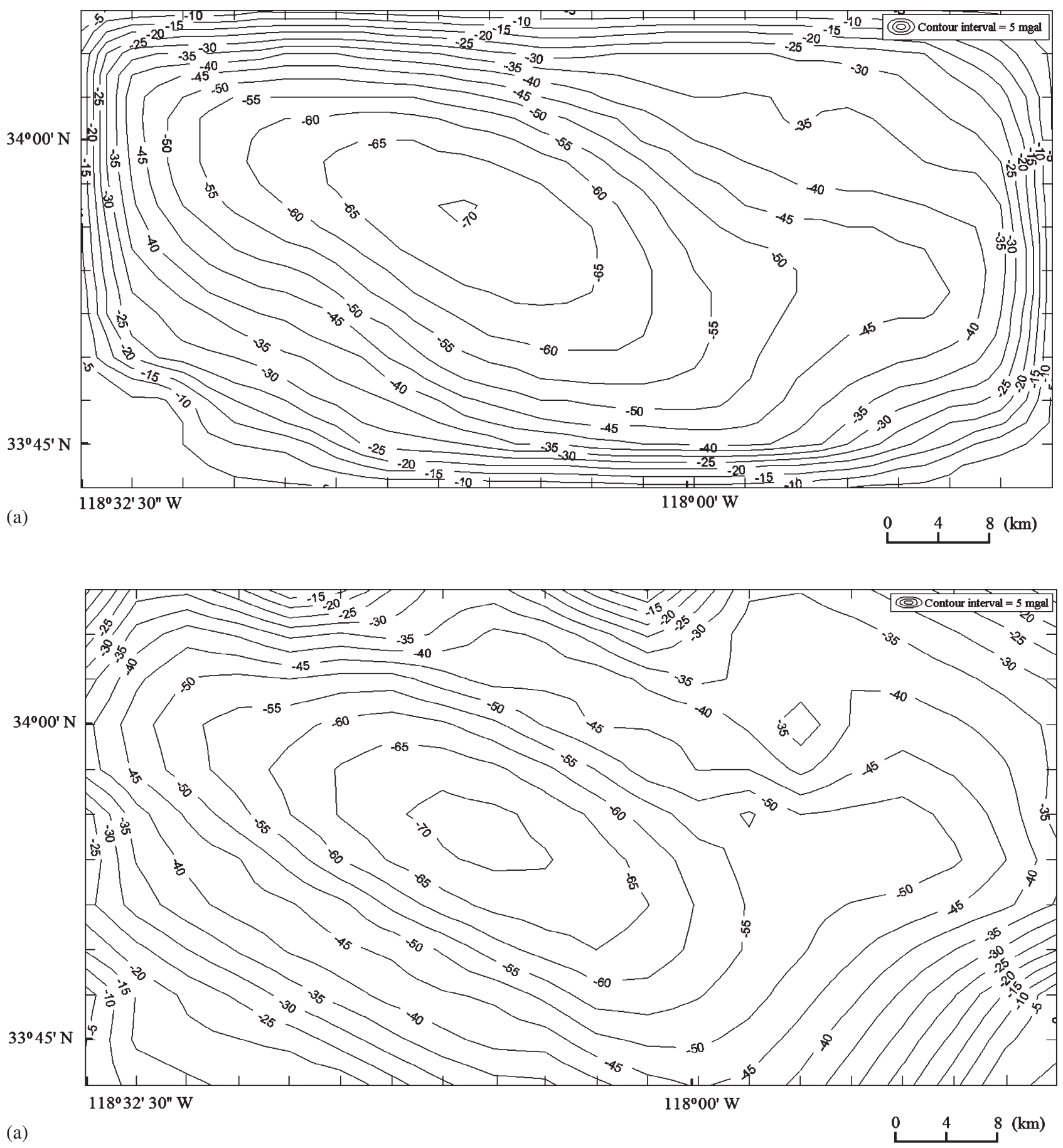

Figure 5. Computed gravity anomaly maps of Los Angeles basin, California with parabolic density function for $\Delta \rho_{0}=$ $-0.5206 \mathrm{~g} / \mathrm{cm}^{3}, \alpha=0.0576 \mathrm{~g} / \mathrm{cm}^{3} / \mathrm{km}$ (a) using 3D vertical prism with parabolic density function (Chakravarthi et al. 2002) and (b) using our vertical pyramid model with parabolic density function.

Table 1. Error estimates of gravity forward modelling.

\begin{tabular}{|c|c|c|c|c|}
\hline $\begin{array}{lc}\Delta \rho_{0} & \alpha \\
\left(\mathrm{g} / \mathrm{cm}^{3}\right) & \left(\mathrm{g} / \mathrm{cm}^{3} / \mathrm{km}\right)\end{array}$ & $\begin{array}{l}\text { RMS error } \\
\text { between residual } \\
\text { (Chai and Hinze 1988) } \\
\text { and our } \\
\text { computed gravity } \\
\text { anomaly (mgal) }\end{array}$ & $\begin{array}{l}\text { NRMS error } \\
\text { between residual } \\
\text { (Chai and Hinze 1988) } \\
\text { and our } \\
\text { computed } \\
\text { gravity anomaly }\end{array}$ & $\begin{array}{l}\text { RMS error } \\
\text { between residual } \\
\text { (Chai and Hinze 1988) } \\
\text { and computed } \\
\text { gravity anomaly (mgal) } \\
\text { (Chakravarthi et al. 2002) }\end{array}$ & $\begin{array}{l}\text { NRMS error } \\
\text { between residual } \\
\text { (Chai and Hinze 1988) } \\
\text { and computed } \\
\text { gravity anomaly } \\
\text { (Chakravarthi et al. } 2002 \text { ) }\end{array}$ \\
\hline-0.5206 & 7.0599 & 0.1038 & 12.0766 & 0.1776 \\
\hline
\end{tabular}


be noted that Chakravarthi et al. (2002) have not discussed these aspects (Lt $\alpha \rightarrow 0$ ) in their paper, even though such a problem exists in their solution too.

\subsubsection{Coincident coordinates of observation point with vertices of pyramid model}

Case 1: Coincident lateral coordinates of observation point with that of both top and bottom pyramid vertices.

Case 2: Coincident lateral coordinates of top and bottom pyramid vertices.

Based on our numerical simulations, in both cases, the top and bottom vertices of pyramid model corners and observation point have to differ by $5 * 10^{-3}$ $\mathrm{km}$ with each other and the top surface of the pyramid model should be more than 1/200 times of the unit grid interval of datum plane for parabolic density function. In case of constant density contrast it should be more than $1 / 20$ times of the unit grid interval as discussed above (section 3.3.1).

By considering the procedure of Chakravarthi et al. (2002), one needs a minimum of 209 vertical prisms for modelling the Los Angeles basin. For a similar exercise, we need only 9 unit pyramids achieving a better accuracy (table 1) if compared to that of Chakravarthi et al. (2002). If the gravity anomaly pattern is observed closely, one can notice that on the corners of figure $5(\mathrm{~b})$, our model matches well with the standard gravity anomaly pattern of Los Angeles basin (Chai and Hinze 1988, figure 4b) compared to that of Chakravarthi et al. (2002). This may be due to the inadequate meshing of the basin by classical vertical prism model (Chakravarthi et al. 2002).

Thus, our pyramid model (figure 1a) offers a better approximation and ease in implementing gravity forward modelling. For present day computer infrastructure, complicated analytic expressions such as equations (5) and (A5) do not constitute computational hurdles.

\section{Summary and conclusions}

Our theoretical gravity anomaly expression devised for $3 \mathrm{D}$ vertical pyramid model with parabolic density contrast variation with depth is novel. Our gravity expression is validated against that of right rectangular parallelepiped model (Banerjee and Das Gupta 1977). Effect of singularities arising in the validation exercise can be minimized by a proper choice of parameter $\alpha$ and perturbation of vertices of pyramid model with references to that of observation points. We have implemented two synthetic experiments and one case study demonstrate the utility of our forward problem

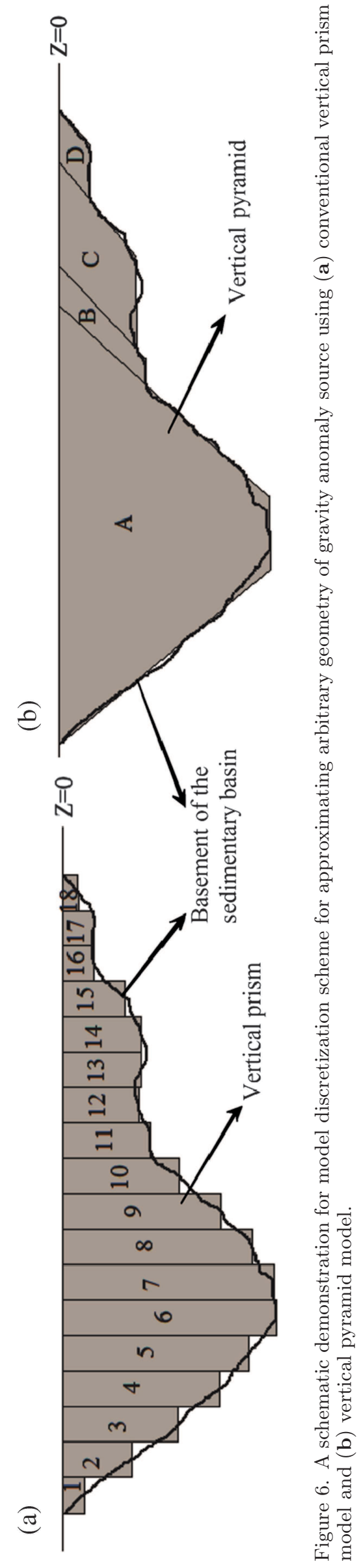


solution. In case study we also have shown the superiority of our model to that of classical vertical prism model (Chakravarthi et al. 2002).

Our pyramid model with its gravity response is an advance in gravity forward modelling and it is quite effective as a building block in comparison to the conventional rectangular parallelepiped model.

The relevant derivations of tensorial gravity for our model are underway.

\section{Acknowledgements}

The integral evaluations in our formulation of gravity forward problem are undertaken by Wolfram Mathematica 9.0.1. Drafting of illustrations in our paper are implemented through MATLAB 2013b. Mr. Anand P Gokula is thankful to Ministry of Human Resources Development (MHRD), Government of India, for financial support.

\section{Appendix}

\section{Expression of the gravity anomaly for pyramid model}

From equations (3 and 4), we can rewrite the parabolic density function $\Delta \rho\left(\zeta^{\prime}\right)$ and the limits $\xi^{\prime}$ and $\eta^{\prime}$ of equation (3) as:

$$
\left.\begin{array}{l}
\Delta \rho\left(\zeta^{\prime}\right)=\Delta \rho_{0}^{3} /\left[r-\alpha \zeta^{\prime}\right]^{2}, \\
r=\Delta \rho_{0}-\alpha\left(z-h_{1}\right), \\
\xi^{\prime}=\xi_{l}^{\prime}-x=m_{1} \zeta^{\prime}+c_{1}, \\
\xi^{\prime}=\xi_{u}^{\prime}-x=m_{2} \zeta^{\prime}+c_{2}, \\
\eta^{\prime}=\eta_{l}^{\prime}-y=m_{3} \zeta^{\prime}+c_{3}, \\
\eta^{\prime}=\eta_{u}^{\prime}-y=m_{4} \zeta^{\prime}+c_{4},
\end{array}\right\}
$$

where

$$
\begin{aligned}
& m_{1}=\left(\xi_{3}-\xi_{1}\right) /\left(h_{2}-h_{1}\right), \\
& m_{2}=\left(\xi_{4}-\xi_{2}\right) /\left(h_{2}-h_{1}\right), \\
& m_{3}=\left(\eta_{3}-\eta_{1}\right) /\left(h_{2}-h_{1}\right), \\
& m_{4}=\left(\eta_{4}-\eta_{2}\right) /\left(h_{2}-h_{1}\right), \\
& c_{1}=\left(h_{1}-z\right)\left(\xi_{1}-\xi_{3}\right) /\left(h_{2}-h_{1}\right)+\xi_{1}-x, \\
& c_{2}=\left(h_{1}-z\right)\left(\xi_{2}-\xi_{4}\right) /\left(h_{2}-h_{1}\right)+\xi_{2}-x, \\
& c_{3}=\left(h_{1}-z\right)\left(\eta_{1}-\eta_{3}\right) /\left(h_{2}-h_{1}\right)+\eta_{1}-y, \\
& c_{4}=\left(h_{1}-z\right)\left(\eta_{2}-\eta_{4}\right) /\left(h_{2}-h_{1}\right)+\eta_{2}-y
\end{aligned}
$$

Then, by performing integration with respect to $\xi^{\prime}$ and $\eta^{\prime}$ in equation (3), we get:

$$
\begin{aligned}
& g_{\text {pyramid }}(x, y, z)=\gamma \Delta \rho_{0}^{3} \int_{\zeta^{\prime}=h_{1}-z}^{h_{2}-z} 1 /\left[r-\alpha \zeta^{\prime}\right]^{2} \\
& \quad \times\left\{\operatorname { t a n } ^ { - 1 } \left[\left(m_{2} \zeta^{\prime}+c_{2}\right)\left(m_{4} \zeta^{\prime}+c_{4}\right)\right.\right. \\
& \left./\left(\zeta^{\prime} \sqrt{\left(m_{2} \zeta^{\prime}+c_{2}\right)^{2}+\left(m_{4} \zeta^{\prime}+c_{4}\right)^{2}+\zeta^{\prime 2}}\right)\right]
\end{aligned}
$$

$$
\begin{aligned}
& -\tan ^{-1}\left[\left(m_{2} \zeta^{\prime}+c_{2}\right)\left(m_{3} \zeta^{\prime}+c_{3}\right)\right. \\
& \left./\left(\zeta^{\prime} \sqrt{\left(m_{2} \zeta^{\prime}+c_{2}\right)^{2}+\left(m_{3} \zeta^{\prime}+c_{3}\right)^{2}+\zeta^{\prime 2}}\right)\right] \\
& -\tan ^{-1}\left[\left(m_{1} \zeta^{\prime}+c_{1}\right)\left(m_{4} \zeta^{\prime}+c_{4}\right)\right. \\
& \left./\left(\zeta^{\prime} \sqrt{\left(m_{1} \zeta^{\prime}+c_{1}\right)^{2}+\left(m_{4} \zeta^{\prime}+c_{4}\right)^{2}+\zeta^{\prime 2}}\right)\right] \\
& +\tan ^{-1}\left[\left(m_{1} \zeta^{\prime}+c_{1}\right)\left(m_{3} \zeta^{\prime}+c_{3}\right)\right. \\
& \left.\left./\left(\zeta^{\prime} \sqrt{\left(m_{1} \zeta^{\prime}+c_{1}\right)^{2}+\left(m_{3} \zeta^{\prime}+c_{3}\right)^{2}+\zeta^{\prime 2}}\right)\right]\right\} d \zeta^{\prime} .
\end{aligned}
$$

As the integration of terms on RHS of equation (A3) is an involved job, we undertake the integration task in a systematic manner. Wolfram Mathematica 9.0.1 is used for carrying out integration. A cursory look at equation (A3) reveals that RHS have four terms. Here, we include the result of integration for the first terms of the RHS part of equation (A3).

$$
\begin{aligned}
& g_{1}(x, y, z)=\int_{h_{1}-z}^{h_{2}-z} 1 /\left[r-\alpha \zeta^{\prime}\right]^{2} \\
& \quad \times\left\{\operatorname { t a n } ^ { - 1 } \left[\left(m_{2} \zeta^{\prime}+c_{2}\right)\left(m_{4} \zeta^{\prime}+c_{4}\right)\right.\right. \\
& \left.\left.\quad /\left(\zeta^{\prime} \sqrt{\left(m_{2} \zeta^{\prime}+c_{2}\right)^{2}+\left(m_{4} \zeta^{\prime}+c_{4}\right)^{2}+\zeta^{\prime 2}}\right)\right]\right\} d \zeta^{\prime} .
\end{aligned}
$$

Upon integration with respect to $\zeta$, we get:

$$
\begin{aligned}
& g_{1}(x, y, z)=-\tan ^{-1}\left[\left(M_{1} \zeta^{\prime 2}+M_{2} \zeta^{\prime}+M_{3}\right) /\left(\zeta^{\prime} R^{\prime}\right)\right] \\
& /\left[\alpha\left(-r+\alpha \zeta^{\prime}\right)\right] \\
& \quad-V_{5} \log \left(-r+\alpha \zeta^{\prime}\right) /\left(2 \sqrt{\alpha^{2} k_{1}+\alpha b_{1} r+a_{1} r^{2}} V_{6}\right) \\
& +V_{5} \log \left(2 \alpha k_{1}+b_{1} r+\alpha b_{1} \zeta^{\prime}+2 a_{1} r \zeta^{\prime}\right. \\
& \left.+2 \sqrt{\alpha^{2} k_{1}+\alpha b_{1} r+a_{1} r^{2}} R^{\prime}\right) \\
& /\left(2 \sqrt{\alpha^{2} k_{1}+\alpha b_{1} r+a_{1} r^{2}} V_{6}\right) \\
& +V_{z 1} /\left(2 \alpha V_{6}\right) \stackrel{h_{2}-z}{h_{1}-z}
\end{aligned}
$$

where

$$
\begin{aligned}
V_{z 1}= & {\left[V _ { 1 } \left(4 \alpha^{3} k_{1} M_{2} M_{3}^{2}-3 \alpha^{3} b_{1} M_{3}^{3}+2 \alpha^{2} k_{1}^{2} M_{3} r\right.\right.} \\
& +2 \alpha^{2} k_{1} M_{2}^{2} M_{3} r+6 \alpha^{2} k_{1} M_{1} M_{3}^{2} r-\alpha^{2} b_{1} M_{2} M_{3}^{2} r \\
& -4 \alpha^{2} a_{1} M_{3}^{3} r+2 \alpha b_{1} k_{1} M_{3} r^{2}+4 \alpha k_{1} M_{1} M_{2} M_{3} r^{2}
\end{aligned}
$$


$+\alpha b_{1} M_{1} M_{3}^{2} r^{2}-2 \alpha a_{1} M_{2} M_{3}^{2} r^{2}+2 a_{1} k_{1} M_{3} r^{3}$

$\left.+2 k_{1} M_{1}^{2} M_{3} r^{3}\right)+V_{2}\left(2 \alpha^{3} k_{1}^{2} M_{3}\right.$

$+2 \alpha^{3} k_{1} M_{2}^{2} M_{3}+6 \alpha^{3} k_{1} M_{1} M_{3}^{2}-\alpha^{3} b_{1} M_{2} M_{3}^{2}$

$-4 \alpha^{3} a_{1} M_{3}^{3}+5 \alpha^{2} b_{1} k_{1} M_{3} r$

$+8 \alpha^{2} k_{1} M_{1} M_{2} M_{3} r+\alpha^{2} b_{1} M_{2}^{2} M_{3} r$

$+7 \alpha^{2} b_{1} M_{1} M_{3}^{2} r-10 \alpha^{2} a_{1} M_{2} M_{3}^{2} r$

$+3 \alpha b_{1}^{2} M_{3} r^{2}+2 \alpha a_{1} k_{1} M_{3} r^{2}+2 \alpha k_{1} M_{1}^{2} M_{3} r^{2}$

$+8 \alpha b_{1} M_{1} M_{2} M_{3} r^{2}-4 \alpha a_{1} M_{2}^{2} M_{3} r^{2}$

$\left.+3 a_{1} b_{1} M_{3} r^{3}+3 b_{1} M_{1}^{2} M_{3} r^{3}\right)$

$+V_{3}\left(2 \alpha^{3} b_{1} k_{1} M_{3}+4 \alpha^{3} k_{1} M_{1} M_{2} M_{3}\right.$

$+\alpha^{3} b_{1} M_{1} M_{3}^{2}-2 \alpha^{3} a_{1} M_{2} M_{3}^{2}+3 \alpha^{2} b_{1}^{2} M_{3} r$

$+2 \alpha^{2} a_{1} k_{1} M_{3} r+2 \alpha^{2} k_{1} M_{1}^{2} M_{3} r$

$+8 \alpha^{2} b_{1} M_{1} M_{2} M_{3} r-4 \alpha^{2} a_{1} M_{2}^{2} M_{3} r$

$-\alpha b_{1} k_{1} M_{1} r^{2}+\alpha b_{1}^{2} M_{2} r^{2}-2 \alpha a_{1} k_{1} M_{2} r^{2}$

$-4 \alpha k_{1} M_{1}^{2} M_{2} r^{2}+3 \alpha b_{1} M_{1} M_{2}^{2} r^{2}$

$-2 \alpha a_{1} M_{2}^{3} r^{2}+7 \alpha a_{1} b_{1} M_{3} r^{2}+5 \alpha b_{1} M_{1}^{2} M_{3} r^{2}$

$+4 \alpha a_{1} M_{1} M_{2} M_{3} r^{2}-2 a_{1} k_{1} M_{1} r^{3}$

$-2 k_{1} M_{1}^{3} r^{3}+a_{1} b_{1} M_{2} r^{3}+b_{1} M_{1}^{2} M_{2} r^{3}$

$\left.+4 a_{1}^{2} M_{3} r^{3}+4 a_{1} M_{1}^{2} M_{3} r^{3}\right)$

$+V_{4}\left(2 \alpha^{3} a_{1} k_{1} M_{3}+2 \alpha^{3} k_{1} M_{1}^{2} M_{3}\right.$

$+3 \alpha^{2} a_{1} b_{1} M_{3} r+3 \alpha^{2} b_{1} M_{1}^{2} M_{3} r-2 \alpha a_{1} k_{1} M_{1} r^{2}$

$-2 \alpha k_{1} M_{1}^{3} r^{2}+\alpha a_{1} b_{1} M_{2} r^{2}+\alpha b_{1} M_{1}^{2} M_{2} r^{2}$

$+4 \alpha a_{1}^{2} M_{3} r^{2}+4 \alpha a_{1} M_{1}^{2} M_{3} r^{2}-a_{1} b_{1} M_{1} r^{3}$

$\left.\left.-b_{1} M_{1}^{3} r^{3}+2 a_{1}^{2} M_{2} r^{3}+2 a_{1} M_{1}^{2} M_{2} r^{3}\right)\right] \underset{h_{1}-z}{h_{2}-z}$

$V_{1}=W_{1}+W_{2}+W_{3}+W_{4}$,

$V_{2}=r_{1} W_{1}+r_{2} W_{2}+r_{3} W_{3}+r_{4} W_{4}$,

$V_{3}=r_{1}^{2} W_{1}+r_{2}^{2} W_{2}+r_{3}^{2} W_{3}+r_{4}^{2} W_{4}$,

$V_{4}=r_{1}^{3} W_{1}+r_{2}^{3} W_{2}+r_{3}^{3} W_{3}+r_{4}^{3} W_{4}$,

$$
\begin{aligned}
W_{1}= & \log \left[\left(\zeta^{\prime}-r_{1}\right) /\left(2 k_{1}+b_{1} \zeta^{\prime}+b_{1} r_{1}\right.\right. \\
& \left.\left.+2 a_{1} \zeta^{\prime} r_{1}+2 R^{\prime} \sqrt{a_{1} r_{1}^{2}+b_{1} r_{1}+k_{1}}\right)\right] \\
& /\left[\sqrt{a_{1} r_{1}^{2}+b_{1} r_{1}+k_{1}}\left(a_{1}+M_{1}^{2}\right)\right. \\
& \left.\times\left(r_{1}-r_{2}\right)\left(r_{1}-r_{3}\right)\left(r_{1}-r_{4}\right)\right]
\end{aligned}
$$

$W_{2}=\log \left[\left(\zeta^{\prime}-r_{2}\right) /\left(2 k_{1}+b_{1} \zeta^{\prime}+b_{1} r_{2}\right.\right.$

$$
\begin{aligned}
& \left.\left.+2 a_{1} \zeta^{\prime} r_{2}+2 R^{\prime} \sqrt{a_{1} r_{2}^{2}+b_{1} r_{2}+k_{1}}\right)\right] \\
& /\left[\sqrt{a_{1} r_{2}^{2}+b_{1} r_{2}+k_{1}}\right]\left(a_{1}+M_{1}^{2}\right) \\
& \left.\times\left(-r_{1}+r_{2}\right)\left(r_{2}-r_{3}\right)\left(r_{2}-r_{4}\right)\right]
\end{aligned}
$$

$W_{3}=\log \left[\left(\zeta^{\prime}-r_{3}\right) /\left(2 k_{1}+b_{1} \zeta^{\prime}+b_{1} r_{3}\right.\right.$

$$
\begin{aligned}
& \left.\left.+2 a_{1} \zeta^{\prime} r_{3}+2 R^{\prime} \sqrt{a_{1} r_{3}^{2}+b_{1} r_{3}+k_{1}}\right)\right] \\
& /\left[\sqrt{a_{1} r_{3}^{2}+b_{1} r_{3}+k_{1}}\left(a_{1}+M_{1}^{2}\right)\right. \\
& \left.\times\left(-r_{1}+r_{3}\right)\left(-r_{2}+r_{3}\right)\left(r_{3}-r_{4}\right)\right]
\end{aligned}
$$

$W_{4}=\log \left[\left(\zeta^{\prime}-r_{4}\right) /\left(2 k_{1}+b_{1} \zeta^{\prime}+b_{1} r_{4}\right.\right.$

$$
\begin{aligned}
& \left.\left.+2 a_{1} \zeta^{\prime} r_{4}+2 R^{\prime} \sqrt{a_{1} r_{4}^{2}+b_{1} r_{4}+k_{1}}\right)\right] \\
& /\left[\sqrt{a_{1} r_{4}^{2}+b_{1} r_{4}+k_{1}}\left(a_{1}+M_{1}^{2}\right)\right. \\
& \left.\times\left(-r_{1}+r_{4}\right)\left(-r_{2}+r_{4}\right)\left(-r_{3}+r_{4}\right)\right]
\end{aligned}
$$

$V_{5}=2 \alpha^{3} k_{1} M_{3}+3 \alpha^{2} b_{1} M_{3} r-2 \alpha k_{1} M_{1} r^{2}$

$$
+\alpha b_{1} M_{2} r^{2}+4 \alpha a_{1} M_{3} r^{2}-b_{1} M_{1} r^{3}+2 a_{1} M_{2} r^{3}
$$

$V_{6}=\alpha^{4} M_{3}^{2}+2 \alpha^{3} M_{2} M_{3} r+\alpha^{2} k_{1} r^{2}+\alpha^{2} M_{2}^{2} r^{2}$

$+2 \alpha^{2} M_{1} M_{3} r^{2}+\alpha b_{1} r^{3}+2 \alpha M_{1} M_{2} r^{3}$

$+a_{1} r^{4}+M_{1}^{2} r^{4}$

$r_{1}=-p_{2} /\left(4 p_{1}\right)-s_{1} / 2-\sqrt{s_{2}-s_{3}} / 2$

$r_{2}=-p_{2} /\left(4 p_{1}\right)-s_{1} / 2+\sqrt{s_{2}-s_{3}} / 2$

$r_{3}=-p_{2} /\left(4 p_{1}\right)+s_{1} / 2-\sqrt{s_{2}+s_{3}} / 2$

$r_{4}=-p_{2} /\left(4 p_{1}\right)+s_{1} / 2+\sqrt{s_{2}+s_{3}} / 2$

$s_{1}=\sqrt{p_{2}^{2} /\left(4 p_{1}^{2}\right)-2 p_{3} /\left(3 p_{1}\right)+2^{1 / 3}\left(p_{3}^{2}-3 p_{2} p_{4}+12 p_{1} p_{5}\right) /\left(3 p_{1} s\right)+s /\left(3 \times 2^{1 / 3} p_{1}\right)}$

$s_{1}=p_{2}^{2} /\left(2 p_{1}^{2}\right)-4 p_{3} /\left(3 p_{1}\right)-2^{1 / 3}\left(p_{3}^{2}-3 p_{2} p_{4}+12 p_{1} p_{5}\right) /\left(3 p_{1} s\right)-s /\left(3 \times 2^{1 / 3} p_{1}\right)$

$s_{3}=\left(-p_{2}^{3} / p_{1}^{3}+4 p_{2} p_{3} / p_{1}^{2}-8 p_{4} / p_{1}\right) /\left(4 s_{1}\right)$

$s=\left[2 p_{3}^{3}-9 p_{2} p_{3} p_{4}+27 p_{1} p_{4}^{2}+27 p_{2}^{2} p_{5}-72 p_{1} p_{3} p_{5}\right.$

$$
\left.+\sqrt{-4\left(p_{3}^{2}-3 p_{2} p_{4}+12 p_{1} p_{5}\right)^{3}+\left(2 p_{3}^{3}-9 p_{2} p_{3} p_{4}+27 p_{1} p_{4}^{2}+27 p_{2}^{2} p_{5}-72 p_{1} p_{3} p_{5}\right)^{2}}\right]^{1 / 3}
$$

$a_{1}=m_{2}^{2}+m_{4}^{2}+1, \quad b_{1}=2\left(m_{2} c_{2}+m_{4} c_{4}\right), \quad k_{1}=c_{2}^{2}+c_{4}^{2}, \quad R^{\prime}=\sqrt{a_{1} \zeta^{\prime 2}+b_{1} \zeta^{\prime}+k_{1}}$,

$M_{1}=m_{2} m_{4}, \quad M_{2}=m_{2} c_{4}+m_{4} c_{2}, \quad M_{3}=c_{2} c_{4}$,

$p_{1}=a_{1}+M_{1}^{2}, \quad p_{2}=2 M_{1} M_{2}+b_{1}, \quad p_{3}=2 M_{1} M_{3}+M_{2}^{2}+k_{1}, \quad p_{4}=2 M_{2} M_{3}, \quad p_{5}=M_{3}^{2}$. 
Rest of the integrations on the RHS of equation (A3) can be handled easily by considering expressions (A4) with necessary changes as per the following details:

For $g_{2}(x, y, z)$ :

$$
\begin{aligned}
& a_{1}=m_{2}^{2}+m_{3}^{2}+1, \quad b_{1}=2\left(m_{2} c_{2}+m_{3} c_{3}\right), \\
& k_{1}=c_{2}^{2}+c_{3}^{2}, \quad M_{1}=m_{2} m_{3}, \\
& M_{2}=m_{2} c_{3}+m_{3} c_{2}, \quad M_{3}=c_{2} c_{3},
\end{aligned}
$$

For $g_{3}(x, y, z)$ :

$$
\begin{aligned}
& a_{1}=m_{1}^{2}+m_{4}^{2}+1, \quad b_{1}=2\left(m_{1} c_{1}+m_{4} c_{4}\right), \\
& k_{1}=c_{1}^{2}+c_{4}^{2}, \quad M_{1}=m_{1} m_{4}, \\
& M_{2}=m_{1} c_{4}+m_{4} c_{1}, \quad M_{3}=c_{1} c_{4},
\end{aligned}
$$

For $g_{4}(x, y, z)$ :

$$
\begin{aligned}
& a_{1}=m_{1}^{2}+m_{3}^{2}+1, \quad b_{1}=2\left(m_{1} c_{1}+m_{3} c_{3}\right), \\
& k_{1}=c_{1}^{2}+c_{3}^{2}, \quad M_{1}=m_{1} m_{3}, \\
& M_{2}=m_{1} c_{3}+m_{3} c_{1}, \quad M_{3}=c_{1} c_{3} .
\end{aligned}
$$

Then, the final expression for gravity effect of proposed pyramid model with depth-wise parabolic density contrast, works out to be

$$
g_{\text {pyramid }}(x, y, z)=\gamma \Delta \rho_{0}^{3}\left(g_{1}-g_{2}-g_{3}+g_{4}\right) .
$$

where $g_{1}, g_{2}, g_{3}$ and $g_{4}$ are the gravity expressions of all terms on RHS of equation (A3).

\section{References}

Banerjee B and Das Gupta S P 1977 Short note: Gravitational attraction of a rectangular parallelepiped; Geophysics 42 1053-1055.

Chai Y and Hinze W J 1988 Gravity inversion of an interface above which the density contrast varies exponentially with depth; Geophysics 53 837-845.

Chakravarthi V, Raghuram H M and Singh S B 2002 Short note: 3D forward modelling of basement interfaces above which the density contrast varies continuously with depth; Comput. Geosci. 28 53-57.

D'Urso M G 2013 On the evaluation of the gravity effects of polyhedral bodies and a consistent treatment of related singularities; J. Geodesy 87 239-252.

D'Urso M G 2014a Analytical computation of gravity effects for polyhedral bodies; J. Geodesy 88 13-29, doi: 10.1007/s00190-013-0664-x.

D'Urso M G 2014b Gravity effects of polyhedral bodies with linearly varying density; Cel. Mech. Dyn. Astron. 120 349-372, doi: 10.1007/s10569-014-9578-z.

D'Urso M G 2015 The gravity anomaly of a 2D polygonal body having density contrast given by polynomial functions; Surv. Geophys. 36 391-425.
García-Abdeslem J 1992 Gravitational attraction of a rectangular prism with depth dependent density; Geophysics 57 470-473.

García-Abdeslem J 2005 Gravitational attraction of a rectangular prism with density varying with depth following a cubic polynomial; Geophysics $\mathbf{7 0}$ J39-J42, doi: 10.1190/1.2122413.

Hamayun, Prutkin I and Tenzer 2009 The optimum expression for the gravitational potential of polyhedral bodies having a linearly varying density distribution; J. Geodesy 83 1163-1170, doi: 10.1007/s00190-009-0334-1.

Hansen R O 1999 Short note: An analytical expression for the gravity field of a polyhedral body with linearly varying density; Geophysics 64 75-77.

Holstein H 2002 Gravimagnetic similarity in anomaly formulas for uniform polyhedral; Geophysics 67 1126-1133.

Holstein H 2003 Gravimagnetic anomaly formulas for polyhedral of spatially linear media; Geophysics 68 157-167, doi: $10.1190 / 1.1543203$.

Holstein H, Fitzgerald D J and Stefanov H 2013 Gravimagnetic similarity for homogeneous rectangular prisms; 75 th EAGE Conference \& Exhibition London, UK, 10-13, doi: 10.3997/2214-4609.20130590.

Kwok Y K 1991 Singularities in gravity computation for vertical cylinders and prisms; Geophys. J. Int. 104 1-10.

Martins C M, Barbosa V C F and Silva J B C 2010 Simultaneous 3D depth-to-basement and density-contrast estimates using gravity data and depth control at few points; Geophysics 75 I21-I28, doi: 10.1190/1.3380225.

Nagy D 1966 The gravitational attraction of a right rectangular prism; Geophysics XXX 362-371.

Okabe M 1979 Analytical expressions for gravity anomalies due to homogeneous polyhedral bodies and translation into magnetic anomalies; Geophysics 44 730-741.

Oliveira V C J and Barbosa V C F 2013 3-D radial gravity gradient inversion; Geophys. J. Int. 195(2) 883-902, doi: $10.1093 / \mathrm{gji} / \mathrm{ggt} 307$.

Petrović S 1996 Determination of the potential of homogeneous polyhedral bodies using line integrals; J. Geodesy 71 44-52.

Pohanka V 1988 Optimum expression for computation of the gravity field of a homogeneous polyhedral body; Geophys. Prospect. 36 733-751.

Pohanka V 1998 Optimum expression for computation of the gravity field of a polyhedral body with linearly increasing density; Geophys. Prospect. 46 391-404.

Rao C V, Chakravarthi V and Raju M L 1993 Parabolic density function in sedimentary basin modelling; Pure Appl. Geophys. 140 493-501.

Rao C V, Raju M L and Chakravarthi V 1995 Gravity modelling of an interface above which the density contrast decreases hyperbolically with depth; Appl. Geophys. 34 63-67.

Starostenko V I 1978 Inhomogeneous four-cornered vertical pyramid with flat top and bottom surface, in stable computational method in gravimetric problems (Navukova Dumka, Kiev, Russia), pp. 90-95 (in Russian).

Talwani M and Ewing M 1960 Rapid computation of gravitational attraction of three-dimensional bodies of arbitrary shape; Geophysics XXV 203-225.

Tsoulis D 2000 A note on the gravitational field of the right rectangular prism; Boll. Geod. Sc. Aff. LIX 1 21-35.

Tsoulis D and Petrović S 2001 On the singularities of the gravity field of a homogenous polyhedral body; Geophysics 66 535-539. 